\title{
JHK photometry of Nova V723 Cassiopeiae 1995
}

\author{
U.S. Kamath and N.M. Ashok
}

Astronomy \& Astrophysics Division, Physical Research Laboratory, Navarangpura, Ahmedabad 380009, India

e-mail: kamath@prl.ernet.in, ashok@prl.ernet.in

Received August 10; accepted December 7, 1998

\begin{abstract}
We discuss the evolution of nova V723 Cassiopeiae 1995 based on JHK photometry covering a period of 15 months beginning two months following outburst. The first two post-outburst flares are covered by these observations.
\end{abstract}

Key words: stars: novae, cataclysmic variables - infrared: stars — stars: individual: V723 Cas

\section{Introduction}

Nova Cassiopeiae 1995 (=V723 Cas) was discovered by M. Yamamoto on 24 August 1995 (Hirosawa 1995). Spectroscopic confirmation of the nova was reported soon after (Ohshima et al. 1995; Iijima \& Rosino 1995; Della Valle et al. 1995). The unusual spectroscopic and photometric features of this nova prompted a comparison with the symbiotic nova PU Vul (Munari et al. 1995). However, continued spectroscopic observations affirmed that it was a slow classical nova (Ijima \& Rosino 1996; Ijima et al. 1998). The visual light curve of this nova has a striking resemblance to that of the unusual slow nova HR Del. Near-IR light curves of V723 Cas in its early phase are given by Munari et al. (1996). The first IR observations of this nova reported by Woodward et al. (1995) show that it was in the pseudophotospheric phase. Our observations reveal the persistence of the pseudophotosphere for a long time after outburst (see Sect. 3.3).

\section{JHK photometry}

$J H K$ photometry of V723 Cas was obtained by using a $\mathrm{LN}_{2}$-cooled InSb photometer at the cassegrain focus of the $1.2 \mathrm{~m}$ telescope of PRL's Mt. Abu Infrared Observatory. The details of the experimental setup are given in Ashok et al. (1994). A chopping frequency of $10 \mathrm{~Hz}$, a throw of

Send offprint requests to: U.S. Kamath $30^{\prime \prime}$ and a $26^{\prime \prime}$ aperture was generally used for the observations. A $39^{\prime \prime}$ aperture was used on some occasions and the chopper throw was adjusted to $40^{\prime \prime} . \psi$ Cas $(J=3.01$, $H=2.48, K=2.41$, Varricatt et al. 1998) was used as the standard star for obtaining the nova magnitudes. Two different dewars were used for the observations - dewar 1 was used from Nov. 1996 onwards, and dewar 2 was used for the earlier ones. Our experience with both the systems shows that they are essentially identical. The photometry is accurate to $\pm 0.05 \mathrm{mag}$. The observations, presented in Table 1, cover a period of fifteen months starting from two months since outburst.

\section{Analysis}

\subsection{Visual light curve}

The immediate post-outburst light curves have been presented by Ohsima et al. (1996) and Munari et al. (1996). An up to date coverage of the light curve can be found at The Astronomer WWW site ${ }^{1}$. The nova underwent at least five major brightenings or flares in the post-outburst phase, mimicing the light curve of the slow nova HR Del. The pitfalls in determining the $t_{3}$ timescales of novae with fluctuations during decline and the associated large errors in the estimated absolute magnitude and distance have been discussed by Robb \& Scarfe (1995) in connection with nova PW Vul. In the case of HR Del widely different $t_{3}$ values of 563 and 225 days have been deduced by Sanyal (1974) and Rafanelli \& Rosino (1978) respectively, giving rise to a difference of 2 magnitudes in the derived absolute magnitudes. Here we show that nova V723 Cas is another such nova where the conventional $M_{V}-t_{3}$ relations give a large spread in the derived results. The nova was around 8.8 magnitude in the immediate post-outburst stage before the first flare. We believe that this is the true maximum of the nova. The nova lightcurve has sharp rise and falls during the flares, making it uncharacteristic of the overall decline during those periods. Assuming that the

\footnotetext{
1 http://www.demon.co.uk/astronomer
} 
Table 1. $J H K$ photometry of V723 Cas

\begin{tabular}{lrlll}
\hline \hline Date & JD & $J$ & $H$ & $K$ \\
UT & $2450000+$ & & & \\
\hline Oct. 1995 & & & & \\
25.75 & 16.25 & 6.93 & 7.32 & 6.99 \\
26.70 & 17.20 & 7.37 & 7.04 & 6.87 \\
27.75 & 18.25 & 7.49 & 7.13 & 6.57 \\
31.90 & 22.40 & 7.25 & 7.04 & 6.82 \\
Nov. 1995 & & & & \\
11.89 & 33.39 & 7.29 & 7.16 & 6.84 \\
12.80 & 34.30 & 7.38 & 7.11 & 6.91 \\
13.63 & 35.13 & 7.26 & 7.24 & 6.92 \\
14.74 & 36.24 & 8.12 & 7.54 & 6.76 \\
15.76 & 37.26 & 7.19 & - & 6.89 \\
Dec. 1995 & & & & \\
05.76 & 57.26 & 7.33 & 7.02 & 6.58 \\
06.75 & 58.25 & 7.40 & 7.06 & 7.07 \\
09.74 & 61.24 & 7.33 & 7.04 & 6.73 \\
10.60 & 62.10 & 7.05 & - & 6.64 \\
11.62 & 63.13 & 6.97 & 6.93 & 6.66 \\
12.62 & 64.12 & 6.76 & 6.68 & 6.33 \\
13.62 & 65.12 & 6.82 & - & 6.51 \\
23.62 & 75.12 & 6.41 & 6.00 & 5.50 \\
Jan. 1996 & & & & \\
02.77 & 85.27 & 6.89 & 6.52 & 5.96 \\
04.77 & 87.27 & 6.91 & - & 5.97 \\
05.62 & 88.12 & 7.09 & 6.60 & 6.08 \\
07.60 & 90.10 & 7.58 & 7.19 & 6.51 \\
10.60 & 93.10 & 7.13 & 6.83 & 6.13 \\
13.71 & 96.21 & 7.14 & 6.83 & 6.19 \\
31.85 & 113.35 & 8.09 & - & 7.10 \\
Feb. 1996 & & & & \\
02.60 & 116.10 & 7.93 & 7.56 & 6.38 \\
03.61 & 117.11 & 7.72 & - & 7.03 \\
04.61 & 118.11 & 7.60 & 7.50 & 7.11 \\
05.59 & 119.09 & 7.49 & 7.58 & 6.83 \\
Nov. 1996 & & & & \\
09.77 & 397.27 & - & - & 8.88 \\
Dec. 1996 & & & & \\
06.58 & 424.08 & - & - & 8.34 \\
20.58 & 438.08 & - & - & 8.38 \\
Jan. 1997 & & & & \\
15.63 & 464.13 & 8.77 & 9.12 & 8.97 \\
\hline & & & & \\
\hline & & &
\end{tabular}

flares are superposed on a smoothly declining light curve we estimate $t_{3} \sim 650$ days. On the other hand, Chochol \& Pribulla (1997) get a value of 173 days. These values of $t_{3}$ imply $M_{V}$ of -4.5 and -6.7 respectively. The discordant results arise mainly out of the subjectivity in choosing the true maximum of the nova.

\subsection{Luminosity, reddening and distance to the nova}

In view of the above discussion we use the following unconventional method to derive some fundamental parameters in the case of V723 Cas. The nova can be assumed to have an absolute magnitude of $M_{V}=-6 \pm 1$. This
Table 2. Adopted values of temperatures of the two emission sources in V723 Cas during six representative epochs

\begin{tabular}{crcc}
\hline \hline Epoch & $\begin{array}{r}\text { JD } \\
2450000+\end{array}$ & $\begin{array}{c}T_{\mathrm{pp}} \\
\text { Pseudophotosphere }\end{array}$ & $\begin{array}{c}T_{\mathrm{fr}} \\
\text { Free-free }\end{array}$ \\
\hline I & 16.25 & 15000 & 10000 \\
II & 36.24 & 27000 & 10000 \\
III & 75.12 & 10000 & 10000 \\
IV & 96.21 & 7500 & 10000 \\
V & 116.21 & 11000 & 10000 \\
VI & 464.13 & 11000 & 10000 \\
\hline
\end{tabular}

is in accordance with the values for slow novae in general and the published values for HR Del in particular (Duerbeck 1981). Gonzalez-Riestra et al. (1996) have derived a reddening of $E_{B-V}=0.6$ to the nova from the $220 \mathrm{~nm}$ absorption feaature in IUE spectra. This reddening gives a distance of $3.9 \mathrm{kpc}$ to the nova for the above mean absolute magnitude. A lower $E_{B-V}$ value of 0.45 has been derived by Munari et al. (1996) from the equivalent widths of interstellar sodium lines, while Chochol \& Pribulla (1997) give a mean value of 0.57 . The uncertainties in absolute magnitude and reddening quoted above give 2.4 and $7.6 \mathrm{kpc}$ as the limits to the distance of the nova. Thus, the nova has a luminosity range of $0.82-5.210^{4} L_{\odot}$. We may mention here that in a recent paper Ijima et al. (1998) have derived $A_{V}=0.89 \mathrm{mag}$, $M_{V}=-6.1$ and a distance of $2.95 \mathrm{kpc}$ for the nova.

\subsection{Evolution of the nova}

The nova was in the pre-nebular phase at least up to mid-March 1996 (Ijima \& Rosino 1996). Varying emission lines have very little effect on the broadband fluxes during this stage when they are dominated by the continuum light of the nova. $V$ magnitudes temporally close to our observations have been used to get quasi-simultaneous optical-near-IR fluxes. For this purpose we have used the Ohsima et al. (1996) $V$ magnitudes. For the period after Dec. 95 when the same are not available, we have used photoelectric/CCD magnitudes reported in the VSNET network. We use the $E_{B-V}$ value of 0.6 to determine dereddened fluxes from our photometry. The dereddened $V J H K$ fluxes are in general a result of pseudophotospheric and free-free emission. A decomposition of these fluxes in terms of the two components can be done under certain assumptions using the optical light curve and colours (Ohsima et al. 1996) and spectral appearance (Iijima et al. 1998) as guides. The spectral energy distribution (SED; See Fig. 2) shows the pseudophotosphere to be the most dominant component of the fluxes. Therefore, we assume that only $10 \%$ of the flux at $H$ is contributed by the free-free emission from the gaseous envelope. The adopted values of the temperatures of the two components during six representative epochs are given in Table 2. 


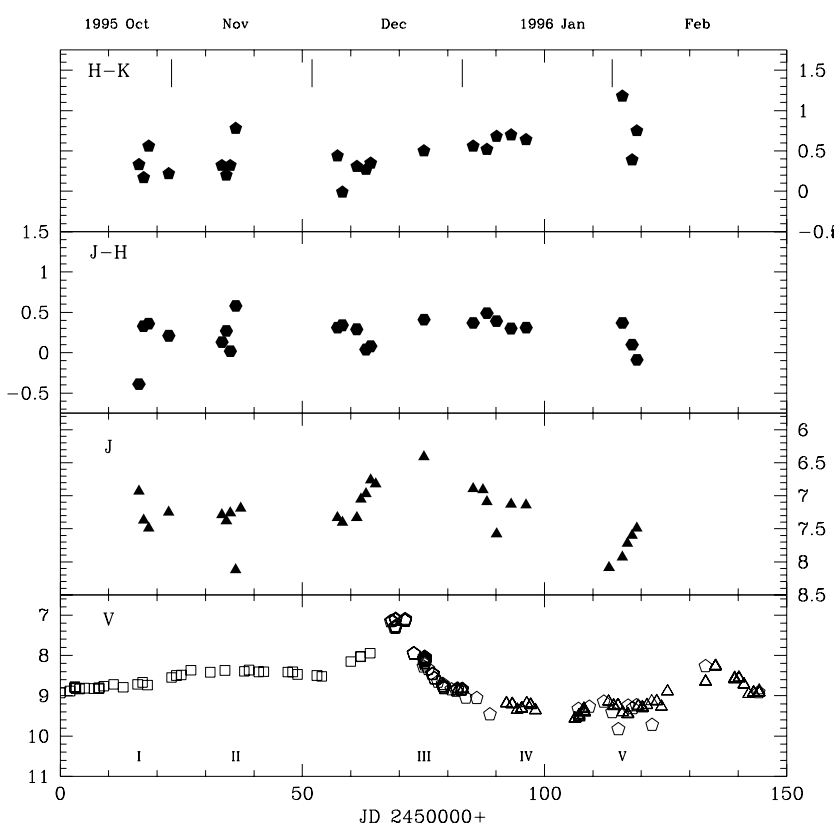

Fig. 1. Optical and IR evolution of nova V723 Cas during the first two flares. $V$ magnitudes have been taken from Ohsima et al. (1996) and data circulated over VSNET. IR data is from Table 1. Epochs I-V are the same as in Fig. 2

This decomposition is not unique, but reasonable changes in the assumptions are sufficient to explain the flux variations over the period of observations.

The first flare and the rising part of the second one have been covered by our observations (see Fig. 1). The nova became brighter in all the three bands, particularly in $J$ and $H$, during the flares. The flaring activity of the nova is a manifestation of episodic mass ejections. Because of this periodic increased mass-loss the pseudophotosphere was able to sustain itself at immediate post-outburst conditions for a long time. Figure 3 shows that the nova lies near other dustless novae in the dereddened two-colour diagram (adapted from Whitelock et al. 1984).

\subsection{Will this nova condense dust?}

This question is prompted both by the expectation of dust condensation in a slow nova and its similarity with HR Del. Infrared data on HR Del during a comparable stage of development are nonexistent and so it is not possible to strictly compare both these novae. The possibility of dust condensation in HR Del has been discussed in the light of IR observations (Geisel et al. 1970), polarization measurements (Zellner 1971) and IRAS data (Harrison \& Gehrz 1988). However, Gallagher (1977) doesnot favour the dust formation scenario in HR Del. No clear evidence of dust formation in V723 Cas is seen in our $J H K$ data. The observed polarization and its variations seem to be
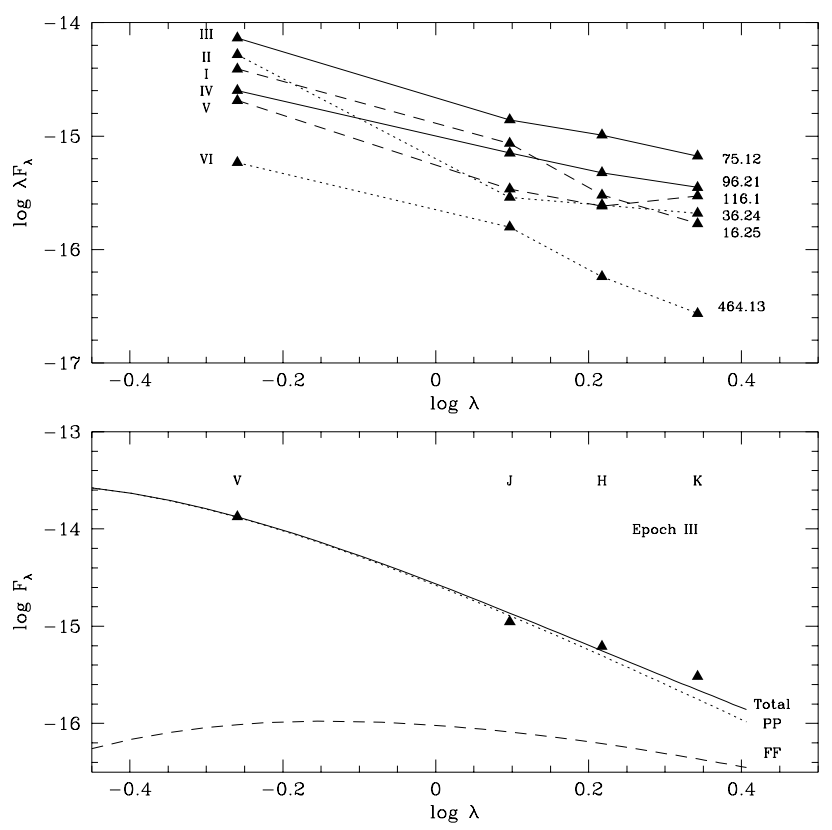

Fig. 2. Top: Dereddened $V J H K$ spectral energy distributions. The epochs are labelled from I-VI and corresponding date (JD-2450000) is written alongside. $F_{\lambda}$ is in units of $\mathrm{W} \mathrm{cm}{ }^{-2} \mu \mathrm{m}^{-1}$ and $\lambda$ is in microns. Connecting lines are drawn as a visual aid. Bottom: The adopted pseudophotosphere (PP) and free-free $(\mathrm{FF})$ spectra for Epoch III are shown by broken curves and their sum (Total) is shown by a soild curve. Dereddened fluxes are also plotted. There is no appreciable excess over the adopted fluxes

because of structural changes in the ejecta rather than because of dust (Johnson et al. 1995, 1996a,b). Continued observations of this nova would help to resolve the puzzle of dust condensation in HR Del-type novae.

\section{Conclusions}

Near-IR photometry of nova V723 Cas undertaken during seventeen months after outburst reveals that its emission was a combination of pseudophotospheric and freefree emission. No evidence of dust formation is seen up to this stage. Episodic mass ejections in the post-outburst period resulted in sharp brightness increases or flares, which were observed in the near-IR also. The nova lies between $2.4-7.6 \mathrm{kpc}$ and has an absolute magnitude of $-6 \pm 1$.

Acknowledgements. Research work at PRL is supported by funds from Dept. of Space, Govt. of India. The authors would like to thank Watson P.V and Anandmayee Tej for help with the observations. We also thank Osamu Ohshima and VSNET observers for communicating the optical observations. Suggestions by Drs. T. Naylor and U. Munari helped to improve the paper. 


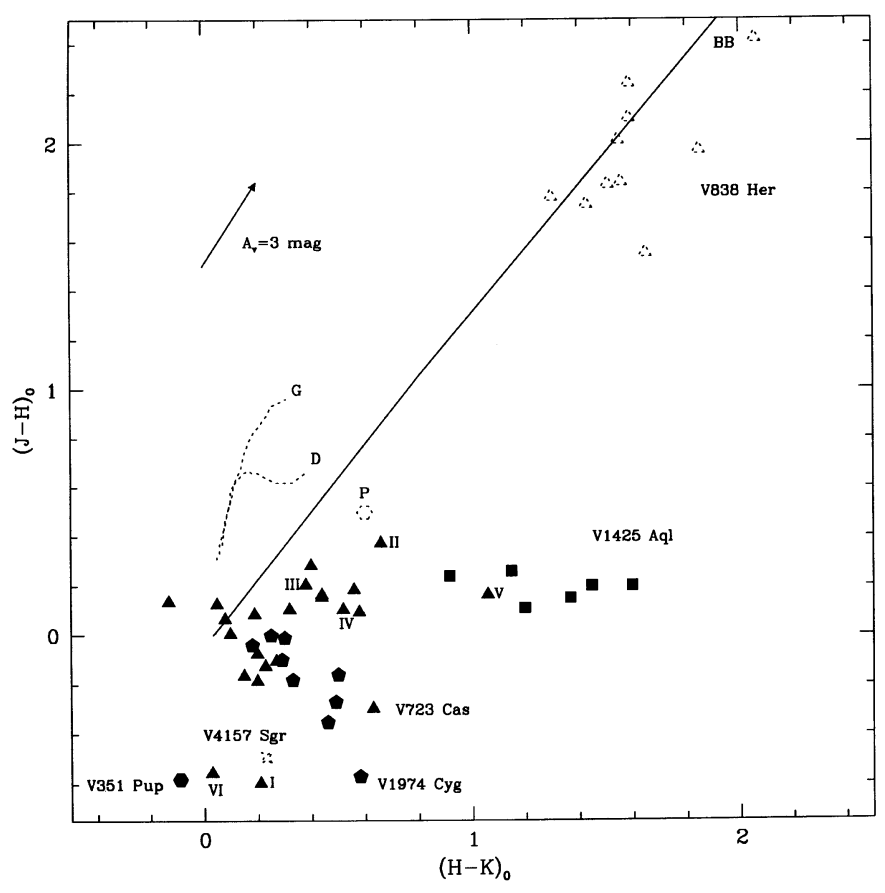

Fig. 3. Two colour plot for novae observed at PRL. The curves for blackbodies (BB), dwarfs (D) and giants (G) and the reddening vector are plotted. The point for a $10^{4} \mathrm{~K}$ plasma is also shown (P). Observed magnitudes of V723 Cas have been dereddened by using $E_{B-V}=0.6$ and are plotted as filled triangles. Roman numerals beside some points denote the epochs as given in Fig. 2. For nova V723 Cas all data points except "VI" are obtained with the same dewar. Other observational points are for V838 Her $\left(A_{V}=0\right.$, Chandrasekhar et al. 1992), V1974 Cyg $\left(A_{V}=0\right.$, Ashok et al. 1992a), V351 Pup $\left(A_{V}=0\right.$, Ashok et al. 1992b), V4157 Sgr $\left(A_{V}=0\right.$, Ashok et al. 1992c) and V1425 Aql $\left(E_{B-V}=0.76\right.$, Kamath et al. 1997). Names of the novae are mentioned beside respective data points

\section{References}

Ashok N.M., Chandrasekhar T., Ragland S., 1992a, IAUC 5525 Ashok N.M., Chandrasekhar T., Ragland S., 1992b, IAUC 5527
Ashok N.M., Chandrasekhar T., Ragland S., 1992c, IAUC 5529 Ashok N.M., Chandrasekhar T., Ragland S., Bhatt H.C., 1994, Exp. Astron. 4, 177

Chandrasekhar T., Ashok N.M., Ragland S., 1992, MNRAS 255, 412

Chochol D., Pribulla T., 1997, Contrib. Astron. Obs. Sk. Pleso 27,53

Della Valle M., Marchiotto W., Lercher G., 1995, IAUC 6214

Duerbeck H.W., 1981, PASP 93, 165

Gallagher J.S., 1977, AJ 82, 209

Geisel S.L., Kleinmann D.E., Low F.J., 1970, ApJ 161, L101

Gonzalez-Riestra R., Shore S.N., Starrfield S., Krautter J., 1996, IAUC 6295

Harrison T.E., Gehrz R.D., 1988, AJ 96, 1001

Hirosawa K., 1995, IAUC 6213

Iijima T., Rosino L., 1995, IAUC 6214

Iijima T., Rosino L., 1996, IAUC 6365

Iijima T., Rosino L., Della Valle M., 1998, A\&A 338, 1006

Johnson J.J., Zellner N.E.B., Ager W., et al., 1995, BAAS 27, 1399

Johnson J.J., Bjorkman K.S., Babler B.L., 1996a, IAUC 6283

Johnson J.J., Bjorkman K.S., Nordsieck K.H., et al., 1996b, BAAS 28, 913

Kamath U.S., Anupama G.C., Ashok N.M., Chandrasekhar T., 1997, AJ 114, 2671

Munari U., Lepardo A., Passuello R., Sostero G., 1995, IAUC 6259

Munari U., Goranskij V.P., Popova A.A., et al., 1996, A\&A 315,166

Ohshima O., Ayani K., Shimuzu M., Yamamoto T., 1995, IAUC 6214

Ohsima O., Akazawa H., Ohkura N., 1996, IBVS 4295

Rafanelli P., Rosino L., 1978, A\&AS 31, 337

Robb R.M., Scarfe C.D., 1995, MNRAS 273, 347

Sanyal A., 1974, ApJS 28, 115

Varricatt W.P., Ashok N.M., Chandrasekhar T., 1998, AJ 116, 1447

Whitelock P.A., Carter B.S., Feast M.W., et al., 1984, MNRAS 211, 421

Woodward C.E., Harker D., Smilowitz J., Rudy R., Kellogg R., 1995, IAUC 6221

Zellner B., 1971, AJ 76, 651 